\title{
LAS POLÍTICAS DE FORMACIÓN DOCENTE
}

\section{José Agustín de Miguel*}

\section{RESUMEN}

Se abordan en este trabajo las políticas de formación docente que comienzan a ser expresas a partir de la formulación del Plan Decenal. Dentro de los lineamientos fundamentales del Plan Decenal, se hizo énfasis en: la capacitación del docente de manera continua, la creación del instituto de profesionalización del magisterio, la formación de los bachilleres maestros y la renovación del currículum de las escuelas normales.

Se partió de la necesidad de realizar un diagnóstico de las instituciones de educación superior y de los profesores de las escuelas normales, con el fin de cualificar la formación de recursos humanos en términos de potenciar un vínculo entre el proceso de aprendizaje enseñanza, enmarcado en el entorno del sistema, como garantía de que la capacitación fuera efectiva.

Entidades como el BID y el Banco Mundial han financiado el Programa de Mejoramiento de la Educación Primaria. La propuesta elaborada en este sentido, sirvió como marco de referencia. tanto para el programa SEEBAC-BID como para el de PRODEP debido a la especificidad de sus planteamientos y a la concreción de las acciones a emprender.

Un diagnóstico de la formación docente delimitaba dos elementos fundamentales de la formación docente: la titulación y la formación del maestro,haciendo hincapié en resultados estadísticos reveladores de la desigual proporción, en términos de mayores esfuerzos invertidos en la titulación de docentes que en la formación de estos.

Las propuestas de formación de los años noventa plantean la concepción de la investigación como herramienta metodológica básica, destacando

\footnotetext{
(") Instituto Tecnológico de Santo Domingo
} 
la dimensión procesual y la potencialización de destrezas cognitivas y afectivas vinculadas a la vida cotidiana del aula para lograr la integración del conocimiento y la experiencia màs cercana a la realidad.

Finalmente, el análisis al marco de referencia legal es imprescindible al momento de las reformulaciones de las politicas de formación docente del magisterio.

l os esfuerzos en este sentido deben estar focalizados de manera conjunta en la resolución de una problemàtica común a todas las instituciones o entidades sociales.

Painaras claves:

Politicas, politicas de formación, politicas curriculares, PRODEP, SLEBAC-BID.

Esta ponencia se refiere mucho más a los planteamientos sobre política que a las acciones que se pudieran derivar de esos planteamientos, porque las acciones caen más dentro del marco de otra exposición que puede analizarlas desde una perspectiva crítica.

Las políticas de formación docente del país realmente coWirnzan a ser expresas a partir del Plan Decenal. También pienso que el Plan Decenal no es solamente una estancia de planificación sino también de transformación o pudiéramos decir que es una estancia de reformas de la educación del país, dentro de ese contexto. Considero que el Plan Decenal implica la toma de conciencia de los problemas, la identificación de las soluciones y la aplicación de aquellas soluciones consideradas como las mejores.

Basándome en esto he dividido la ponencia en tres momentos: la primera estaría marcada por el Plan Educativo y por el Plan Decenal. El Plan Educativo termina con la redacción del famoso decálogo que yo creo que es una reliquia. En ese documento se perciben como problemas de la educación dominicana las deficiencias en la formación, capacitación y actualización del magisterio en todos los niveles. Tenemos ese primer dato. ¿En qué consisten estas deficiencias?. 
Dice el decálogo que los maestros no cuentan con el dominio de las ciencias, de las culturas y de las metodologias para dirigir el proceso de enseñanza-aprendizaje o detallan algunas de las soluciones que en ese momento parecían las más adecuadas. I.as primeras serían:

- el fortalecimiento de las escuelas normales como centru de formación del maestro de primaria:

- la revisión del curriculo, de las metodologías:

- es necesario crear especialidades que actualmente no existen en la formación docente como la tecno-profesional y la educación de adultos;

- la implementación de mecanismos ágiles a bajo costo para la capacitación permanente de los maestros en servicios: y por último

- cursos de capacitación a distancia que permitan satistiacer las necesidades de los docentes.

Obviamente el Plan Educativo tue asumido por el Plan Decenal. En este sentido, el Plan Educativo nunca tuvo la realización de acciones que vinieran a poner o a aplicar las soluciones que se habían identificado, sino que hay un periodo -entre comillas y en el mejor sentido de la palabra- de "confusión" entre lo que era el Plan Educativo y lo que era el Plan Decenal. Hay una mezcla, un traspaso de un proyecto si lo queremos llamar así a otro, sin delimitar claramente cual pertenece a cual, dentro del Plan Decenal.

El Plan Decenal se manejó con las famosas consultas. Si ustedes revisan todo ese material, que es inmenso, van a encontrar una cantidad grande, tanto de edificación de problemas como de soluciones, como de mecanismos para aplicar esas soluciones. Es muy difícil resumir todo ese material, aunque hay un documento que es un intento muy válido. Sin embargo creo que se pueden tomar esos trabajos anteriores y en los documentos del 
Congreso encontramos los lineamientos fundamentales de todo el proceso del Plan Decenal.

Se dice en el Congreso:

1. Declarar la función docente como un elemento contínuo haciendo énfasis en la capacitación y contemplando la modalidad de capacitación a distancia. Es muy importante recordar que en el Plan Educativo se habla de educación a distancia y en el Plan Decenal se vuelve a hablar de educación a distancia.

2. Crear el instituto de profesionalización del magisterio. El profesor normal deberá recibir una formación básica que lo compare profesionalmente a cualquier otro profesional de la educación.

3. La formación de los bachilleres maestros en servicios debe ser una tarea básica.

4. Hacer una renovación del curriculum de las escuelas normales sobre bases más científicas con miras a formar recursos humanos calificados, capaces de vincular el proceso de enseñanza-aprendizaje a la vida. Y aquí tenemos otro elemento muy importante, el cambio, la renovación de las escuelas normales.

Dice: "para que el Plan Decenal alcance los propósitos justos que se propone es preciso realizar un diagnostico de la educación superior y de los profesores de las escuelas normales para utilizar sus resultados en la actualización del magisterio". Ese dato es importante, por lo que después se propone que las escuelas normales sean subidas a nivel superior.

Hay otro dato que yo acabo de entender y es éste. Cada nueve años se deben celebrar cursos de perfeccionamiento cuyos resultados tendrían sus efectos en el escalafón. No se entiende por qué cada nueve años, pero se proponen en el Plan Decenal.

Este dato también es muy importante: cuando veamos los programas del proyecto del mejoramiento de la educación bási- 
ca, nos vamos a encontrar que PRODEP disenina setenta y tantos centros de capacitación por todo el país, SEEBAC/ BID trabaja con un número muy considerable de instituciones también prácticamente a lo largo de todo el país.

Para complementar la formación y capacitación de profesores se debe insistir en la necesidad de formar recursos especializados para la administración, la supervisión y la investigación. Otro elemento importante es no insistir solamente en la capacitación sino relacionarlo con el entorno del sistema para que esa capacitación sea efectiva.

En el Plan Decenal podíamos hacer recuento de los elementos y de las políticas que posteriormente van a sufrir una elaboración mucho más refinada, pero que ya están presentes en el Plan Decenal. Este primer momento pudiera ser complementado con algún otro aporte de otros documentos, me refiero por ejemplo a un pacto por la patria que es producido dentro de una crisis de la Asociación Dominicana de Profesores (ADP) o del magisterio, dentro de una huelga. En ese documento hay dos cosas interesantes:1) se propone un perfil del maestro y 2) se habla de que es necesario un fondo para la capacitación de los maestros. El segundo es un momento del programa de mejoramiento de la educación primaria, es un momento lógico dentro del proceso de reforma de la educación del país.

El Plan Decenal había sido un encuentro entre todas las personas interesadas y no interesadas directamente por la educación que habían planificado y propuesto soluciones, pero era necesario bajar al terreno de la acción, de emprender grandes actividades que fueran respuestas a lo dicho en Plan Decenal y yo creo que ahí está el programa de mejoramiento de la educación primaria, financiado por el Banco Mundial y el BID.

Para estos dos proyectos, si los queremos separar, conforme a los contextos de financiamiento de los dos bancos. se preparó una propuesta para el establecimiento de un nuevo sistema de formación de maestros a nivel básico. Este documento posible- 
mente no haya circulado mucho en el país en ese momento. Sin embargo, fue el marco de referencia tanto para el programa de SEEBAC-BID como para el programa de PRODEP y en él se encuentran elementos de políticas docentes que muy difícilmente hayan sido superados por documentos posteriores. ¿Por qué? Porque tiene planteamientos muy especificos. muy concretos y propone acciones también bastantes reales. Un análisis de ese documento, por el impacto que tuvo en la formulación de los programas, creo que es interesante.

Este documento parte de un principio que es fundamental. En la política de formación docente había que distinguir dos cosas: una la titulación y otra la formación de maestros. ¿Por qué esa afirmación? Porque se tomaban las estadísticas de las características de formación de los maestros y se encontraba la siguiente realidad: que el $47.5 \%$ de los maestros que a la altura del 1993 estaban en las aulas habían sido titulados en programas de emergencias, de poca duración y con elementos muy simples; el 14\% habían sido formados para el nivel medio, pero estaban trabajando en educación básica; el 14.5\% eran bachilleres y solamente el $24 \%$ procedian de las escuelas normales.

Hay que recordar que este $24 \%$ es el porciento de aquellos que el documento considera como formados. Los demás son titulados.

Entonces, a partir de esa realidad, de que se había hecho mucho por titular y muy poco por formar, se presenta una propuesta para hacer un sistema de formación de maestros para nivel básico. Esta propuesta tiene unos objetivos que son válidos ahora como en la fecha en que fueron presentados.

En primer lugar, la Secretaría de Educación debía enviar un mensaje simple y claro a la sociedad dominicana sobre la realidad del magisterio y la necesidad de cambiar esa dirección de titulación por una de formación.

Los otros objetivos eran: 
- establecer un solo paradigma para la formación de maestros;

- retirar de circulación todos aquellos programas que vinieran solamente a titular;

- establecer el bachillerato como conducta de entrada a cualquier escuela de educación, privada o pública:

- establecer la licenciatura como conducta de entrada a la profesión de maestro dominicano de cualquier área o nivel con niveles de transición de dos años para aplicar:

- transformar las escuelas normales en una institución de educación superior capaces de responder al nuevo paradigma (se retoma otra vez la situación de las escuelas normales):

- integrar todas las escuelas de educación del país, públicas y escuelas normales, públicas y privadas en la formación de maestros de todos los niveles educativos:

- la creación de un sistema de incentivos para atracr a buenos estudiantes a la carrera de educación:

- la estructuración de un programa nacional de formación de formadores de maestros de pre-escolar y de primaria; y

- el establecimiento de un sistema nacional de evaluación y acreditación de las escuelas y de exámenes, como requisitos previos para el ejercicio de la profesión de maestros en la escuela pública y privada.

Estos objetivos realmente no se han implementado a plenitud. Se han hecho muchos esfuerzos significativos en la formación de formadores, pero no hemos hecho realmente un sistema nacional de evaluación y acreditación de las escuelas de formación de maestros y tampoco se ha hecho el examen para ejercer en las escuelas.

Dentro de estas propuestas también se propone una lista de postulados de políticas curriculares que son sumamente intere- 
santes. En primer lugar una concepción de la investigación como estrategia metodológica básica con énfasis en las dimensiones procesuales donde se potencien destrezas cognitivas y afectivas vinculadas a los procesos cotidianos del aula con hincapié en lograr una integración de los campos de conocimiento y experiencias que faciliten una comprensión más reflexiva y crítica de la realidad, así como una visión interdisciplinaria de las ciencias que articule la ciencia y la tecnología, que permita la modificación de nuestras representaciones de la realidad social y natural, apoyados por la investigación científica y el desarrollo tecnológico, que reconoce la necesidad de conectar el ambiente experencial con el entorno, que concibe la realidad como un espacio de aprendizaje, que facilita un conocimiento integrado propio de la interdisciplinareidad, donde se destaca el valor de la experiencia como punto de partida del aprendizaje, considerándose como un elemento fundamental e indispensable para la reflexión, que entiende como fundamental que la articulación de la práctica en la teoría estimulará la utilización de procedimientos de indagación en el aprendizaje por descubrimiento y la adquisición de nuevos esquemas de pensamiento y acción.

Esto se fundamenta en la comprensión de cómo se elabora, se produce y transforma el conocimiento, lo que implica que el conocimiento tiene una naturaleza histórica y social que asume la existencia de un saber, que se elabora desde la realidad y desde un saber científico acumulado históricamente, que enfatiza una dimensión ética que favorece el trabajo cooperativo y el trabajo en equipo.

Recuerdo haber visto en muchas aulas todo aquel proceso de supervisión de PRODEP, tanto en los grupos de formación de maestros como en las propias aulas de los maestros cuando íbamos a las escuelas en el 1993. Aquí está el embrión de todo el discurso que se elabora mucho más completo, de transformación curricular y que de ese nivel incipiente de desarrollo pasa a la 
primera gran etapa de formación de maestros que sería SEEBACBIC y PRODEP.

El sistema de formación tienen que ser coherente, innovador, que permita al país enfrentar los retos del desarrollo social y económico; que articule los objetivos de acción, de formación inicial y en servicio; que garantice elevar la calidad de la educación nacional, que permita impulsar a nivel nacional la creación de una nueva escuela.

El perfil que propone es también muy interesante:

- El maestro debe ser dinamizador, orientador y facilitador de situaciones que fomenten el desarrollo de aprendizaje significativo;

- Que proporcione una formación intelectual y moral;

- Creativo, innovador, emprendedor, altamente calificado;

- Practicará la investigación como método de solución de problemas;

- Manejador autónomo y crítico de la información

Aquí tenemos un perfil. PRODEP tenía otro. SEEBAC-BID tenía otro. Y en Transformación Curricular se hizo otro. Hay muchas coincidencias pero hay multiplicidad de perfiles. Este tema del perfil debiera ser todavía manejado, estudiado y estilizado, o sea reducirlo en tamaño, en dimensiones para que fuera mucho más manejable y pudiera ser más efectivamente incorporado a la realidad de la formación de maestros. Esta multiplicidad y disparidad en los perfiles hace que se olviden como marco de referencia cuando se emprenden actividades de formación.

Esta propuesta, como decíamos, fue utilizada para los programas que iban a ser financiados por el Banco Mundial y el BID. Esos programas fueron ejecutados con notable éxito.

La población que asistió a las actividades de esos programas fue significativa. De hecho ahora en el segundo programa no 
hay tantos demandantes de formación a nivel de profesorado. La mayoría se está orientando hacia la licenciatura. La propuesta también marca una modalidad muy interesante y es que propone el programa de 72 créditos, de ahí tienen ustedes de donde se saca el origen tanto de SEEBAC-BIC como de PRODEP.

También hace otra referencia muy interesante y es el porcentaje de los componentes de los planes de estudios. En la propuesta, la formación general debería ocupar el 10\%, las áreas de conocimiento $50 \%$, la formación pedagógica general el 15\% y la formación pedagógica especial el 25\%.

Tanto SEEBAC-BID como PRODEP andan muy cerca de estas cantidades o de estos porcientos con leves variaciones, pero insisto en que fue un marco de referencia para la formulación de los programas. Hay otro referente en esta propuesta que tiene que ser resaltada y es con relación a las escuelas normales.

Parte la propuesta de que la escuela normal necesita una transformación fundamental. ¿En base a qué elemento? El primero, en base a una elevación del nivel como institución de educación superior. Se retoma ese dato en la idea de la concentración en una sola institución de educación superior descentralizada que sea gobernada por una junta con una rectoría de consejo académico.

Para que la formación de maestros sea cualitativamente diferente es importante que se vuelve a tomar a la escuela normal como algo necesario.

Entrando en la tercera etapa de la formulación de la política, hay basicamente dos polos de referencia, uno sería la Ley y el otro sería este documento.

En la Ley, el título 6to. está destinado a la profesionalización, el estatuto y la carrera docente. El artículo 26 afirma: "El Estado dominicano fomentará, garantizará la formación de docentes a nivel superior para la integración al proceso educativo en todos 
los niveles y las distintas modalidades existentes incluyendo el fortalecimiento de centros especializados para tales fines".

Hay una intención de que los docentes sean formados a nivel superior en lugar de a nivel medio como habia sido la tradición del país.

El artículo 129 dice: "Se crea el Instituto Nacional de Formación y Capacitación del Magisterio como organo descentralizado adscrito a la Secretaría de Estado de Educación y Cultura y tendrá como función la coordinación de la oferta de formación. capacitación, actualización y perfeccionamiento del personal de educación en el ámbito nacional". Tenemos otro elemento que había salido previamente. la formación del Instituto.

El artículo 130. "Los estudios magisteriales serán impartidos en el nivel de educación superior en coordinación con el Instituto de Formación y Capacitación del Magisterio“.

Ese podría ser el marco legal que permite. posteriormente. hacer una formulación expresa de la política de formación docente del magisterio dominicano.

El análisis de este documento es fundamental para saber la realidad política que tenemos en los actuales momentos. El documento parte de una definición general cuando dice: "la Secretaría reconoce que contar con un sistema nacional de formación de desarrollo del personal para el sector educativo ofrece la oportunidad de establecer un marco definido de políticas, metas y prioridades creando un espacio propicio para el desarrollo de proyectos, iniciativas concretas de formación que posibiliten la generación de cambios positivos para el logro de niveles cada vez más elevados de eficiencia, calidad y equidad en el sistema”.

En esa declaración se expresa claramente la necesidad de un sistema de formación de personal docente. Hay un dato que justifica esa afirmación. No es posible abordar desde una perspectiva coyuntural la proyección de futuro de aquellas metas y logros 
considerados como fundamentales de acuerdo a las necesidades presentes y futuras de la educación dominicana.

Hoy al final del siglo, se asume la posibilidad de orientar la planificación e implementación de una estrategia global de formación inicial y educación permanente dirigida al personal directivo, docente, técnico y de administración del sistema educativo nacional en el corto, mediano y largo plazo. Hay una intención muy importante: no puede ser el sistema nacional una perspectiva coyuntural en los momentos actuales, dada la importancia que la educación tiene en el país.

En ese sentido, el documento asume la problemática de la formación de profesionales de la educación concitando la participación del Estado y de las entidades de educación superior. Este es otro elemento sumamente valioso.

El sistema es necesario, no es coyuntural y debe ser objeto de esfuerzo, no solamente del Estado, sino de las instituciones privadas de educación superior.

¿Cuáles son las características de ese sistema? Debe ser integral, de globalización permanente, no remedial ni compensatorio. Esas características también son fundamentales, pero además, toda la acción o todas las políticas de perfeccionamiento docente deben incidir en una mejor valorización de los docentes.

¿Por qué es necesario el sistema de formación de docentes?

- porque la educación es hoy día estratégica para el desarrollo,

- porque necesitamos formación de sujetos autónomos y críticos,

- porque se necesitan desarrollar nuevas competencias para el trabajo como creatividad, autonomía, cooperación, capacidad de toma de decisiones, formación en valores, la comprensión y respecto a la diferencia y a la diversidad, 
- porque la educación es una herramienta clave para consolidar la identidad cultural,

- porque es una vía para la superación de la pobreza y los problemas de salud, y

- porque es un factor determinante para la productividad.

Entonces, se percibe al maestro como un mediador entre la escuela y la sociedad.

Este elemento es también un factor nuevo que no había aparecido en ninguno de los documentos anteriores.

Los ejes en el proceso de formación es otro tema que es fundamental y en este sentido propone dos cosas que a mi juicio son las dos deficiencias mayores de todas las acciones anteriores. Dice: “...el sistema de formación de los docentes debe estar enmarcado en la reforma institucional y en la transformación curricular". Creo que ahí no ha habido grandes ni significativos logros en las acciones anteriores.

La meta es que para el año 2005 o 2006 el grado académico mínimo del $80 \%$ del personal sea la licenciatura. Se apoyará para conseguir esto en las escuelas normales y las universidades. Se describe toda una serie de aspectos, como son los grados, el valor del crédito de los programas, etc., etc.

En el año 1998-99 sólo ingresan al sistema los maestros y maestras que hayan completado 15 créditos/semestres del profesorado. A partir del año académico 1999-2000 la Secretaría de Estado de Educación y Cultura (SEEC), sólo contratará los maestros/as de educación inicial o básica que posean el profesorado en educación inicial y básica. A partir del 2000-02 la SEEC sólo contratará los maestros/as de educación inicial que hayan cursado 36 créditos/semestres más allá del profesorado como mínimo.

Yo creo que ahí están las grandes metas del nuevo sistema de formación de maestros. 
¿Cuáles serían algunas de las conclusiones que habria que hacer a lo expuesto en el tema de las políticas de formación docente?

1. La vinculación de la acción de formación de los docentes con Transformación Curricular y con Pruebas Nacionales. Estimo que si no se avanza mucho más en esa vinculación. la formación docente que se pueda hacer en los centros de educación superior y en las escuelas normales no se va a reflejar de una manera eficiente en las aulas de las escuelas de educación básica y van a existir muchas lagunas en esa formación docente que irán incrementando las deficiencias de los maestros y debilitando los logros del sistema. Creo que los maestros debieran tener un mayor conocimiento práctico de lo que significa la Transformación Curricular y ajustar la filosofía de las Pruebas Nacionales a lo que realmente son. utilizar la información generada en las Pruebas Nacionales para el diseño de su planificación en el aula.

No considero que todas estas actividades de Transformación Curricular tengan que ser necesariamente realizadas directamente por el personal. creo que debiera ser también incrementada esa formación y abrir caminos de nuevas acciones de integración del nuevo currículo a la práctica de los maestros en los centros de formación. Con esto vinculo la segunda conclusión.

2. Me da la impresión que aunque se menciona, no se ha hecho lo suficiente en el área de formación de formadores. Tengo la impresión de que no ha habido una transformación profesional ni en la mentalidad, ni en la cultura de la formación o de los formadores. Eso no quiere decir que no haya excelentes formadores de maestros, pero no creo que existan en la cantidad suficiente como para emprender esa gran tarea.

3. Junto con esto, yo también estimo una deficiencia de la cual se ha hablado muy en secreto y que podría ser formulada en un tono especial y es aquella que dice que el Plan Decenal no tuvo una bibliografía. Yo creo que a la politica de formación de 
maestros le hace falta una gran renovación bibliográfica. L.o digo porque me ha tocado visitar las bibliotecas de las escuelas de los centros donde se está haciendo ahora formación de maestros y realmente asusta un poco ver la bibliografía que existe en esos centros.

Creo que no es la que corresponde al paradigma que hay implícito en la política de formación de maestros. Es más, tengo la impresión de que para muchos docentes no es necesaria esa bibliografía. Fíjense que esto estaría íntimamente vinculado con la formación de formadores que debian ser los que presentaran esa necesidad y abrieran pistas.

No creo que solamente sea asunto de los financiamientos de los centros de formación de maestros, creo que tampoco hay una percepción clara de la renovación bibliográfica de nuestro centro de formación para ejecutar el paradigma que implica la formación de maestros.

4. Otro asunto que es fundamental es el siguiente: la real transformación de las escuelas normales. Muy crudamente se podría afirmar que eso de que son de educación no es producto de un decreto sino que implica un proceso de reorganización. sobre todo de un cambio cultural que yo hasta estos momentos no lo he visto.

Posiblemente no haya tenido la oportunidad de verificarlo con todos los elementos de juicio necesarios, pero por el contacto que he tenido con las escuelas normales. es urgente y necesario avanzar mucho más rápida y profundamente en la transformación cultural de las escuelas normales. sobre todo si se llega a tomar la decisión de que ofrezcan y otorguen la licenciatura a los maestros. Sé que se han hecho esfuerzos por la selección de los profesores y he tenido contactos en ese sentido. pero no es suficiente.

5. Otro tema que es vital para la aplicación de la formación de ese paradigma de formación docente. es llevar a una realidad 
la descentralización del sistema, en concreto a las direcciones regionales. Si ustedes hubieran vivido toda la experiencia de visitar prácticamente el país por distrito en la época de PRODEP, se hubieran encontrado como que no estaban muy enterados a nivel de distritos y regionales, sobre lo que se estaba haciendo. Ellos deben tener más participación en la gestión de la formación de los maestros. Una participación real en esas tareas es fundamental.

6. Por último, considero que es fundamental la creación del Instituto Nacional de Formación Docente. Creo que esa instancia se hace ya necesaria para coordinar, marcar pautas, hacer mucho más explícito el documento, hacer avanzar los programas y sobre todo para hacer dos cosas. Además de la formación de formadores, desarrollar investigación que no tenemos y que es necesaria. Sin información es imposible hacer cosas que cualitativamente nos vayan a hacer crecer.

Creo que el Instituto también debe trabajar con las instituciones que están envueltas en la formación de maestros. No dejárselo solo al Consejo Nacional de Educación Superior (CONES). El Instituto debiera ser el interlocutor de transformación de las escuelas de educación que tenemos actualmente. 